\title{
Effect of Inoculation with Arbuscular Mycorrhizal Fungi on Selected Spring Wheat Lines
}

\author{
Yonaisy Mujica Pérez ${ }^{1}$, Christiane Charest ${ }^{3}$, Yolande Dalpé ${ }^{2}$, Sylvie Séguin ${ }^{2}$, Xuelian Wang ${ }^{2} \&$ Shahrokh \\ Khanizadeh $^{2}$ \\ ${ }^{1}$ Instituto Nacional de Ciencias Agrícolas, San José de las Lajas, Cuba \\ ${ }^{2}$ Ottawa Research and Development Centre, Agriculture and Agri-Food Canada, Ottawa, ON, Canada \\ ${ }^{3}$ Department of Biology, Faculty of Science, University of Ottawa, Ottawa, ON, Canada \\ Correspondence: Shahrokh Khanizadeh, Ottawa Research and Development Centre, Agriculture and Agri-Food \\ Canada, Ottawa, ON, Canada. E-mail: Shahrokh.Khanizadeh@agr.gc.ca, http://khanizadeh.info
}

Received: May 31, $2016 \quad$ Accepted: June 22, $2016 \quad$ Online Published: September 11, 2016

doi:10.5539/sar.v5n4p24 URL: http://dx.doi.org/10.5539/sar.v5n4p24

\begin{abstract}
An experiment was performed in a completely randomized split-plot design using five lines of spring wheat (Triticum aestivum L.) (AW-774, AC Carberry, HY-162, Major and AAC Scotia) and two arbuscular mycorrhizal fungi (AMF) strains (Rhizoglomus irregulare and Glomus cubense). Two different inoculant forms (solid and liquid) for the $G$. cubense strain were evaluated. The main plot was AMF, and the subplot was spring wheat lines. Data on heading date, plant height, fresh, and dry biomass, yield, grain quality (chemical composition of the seeds, gluten, and sugar), root structure, and colonization by AMF were collected. The results show a positive effect of inoculation in comparison with the control treatment. The liquid and solid G. cubense inoculants provided better results than inoculation with $R$. irregulare. Fungus indicators were in agreement with root morphological parameters because of the effect induced by AMF activity. Yield increased significantly in the mycorrhizal treatments.
\end{abstract}

Keywords: fungus, colonization, yields, cereal

\section{Introduction}

Wheat (Triticum aestivum L.) is the world's third produced cereal after maize and rice. It is estimated that by 2015 , the production of this cereal will increase by $70 \%$ (Tilman et al., 2011) because of the growing needs of the human population for food, feed, fibre, and fuel (Fedoroff et al., 2010). To meet this demand, and because of the limited availability of uncultivated land (Garnett et al., 2013), agriculture faces a problem related to the transformation of many ecosystems to intensive grain production (Mueller et al., 2012).

Strategies such as the use of more environmentally friendly alternatives, natural processes, and environmental conservation play a vital role in current agricultural production. A large number of microorganisms exert positive effects on the growth and development of plants in the rhizosphere region, and are involved in various activities, including dynamic resources availability to plants and preservation of soil fertility (Priyadharsini \& Muthukumar, 2015).

Microorganisms play an important role in agricultural systems. Specifically, arbuscular mycorrhizal fungi (AMF) are potential components of sustainable management systems (Adesemoye \& Kloepper, 2009). These fungi are biotrophic because they depend on their host to complete their life cycle. They establish symbiotic associations with the majority of terrestrial plants in a diversity of ecosystems (Graham, 2008; Smith \& Read, 2008; Neumann \& George, 2010), improve plant tolerance to both biotic stresses (e.g., pathogens) and abiotic stresses (e.g., drought, soil salinity, and pollution) (Banuelos et al., 2014; Ruiz-Lozano et al., 2012; Cicatelli et al., 2014; Songachan et al., 2011), and play a role in remediation (Mrnka et al., 2012).

Over the past few decades, companies throughout the world have manufactured and commercialized AMF inoculants using either single AMF species or mixtures of AMF species that may include plant-growth-promoting rhizobacteria or other symbiotic and/or biocontrol fungi (Gianinazzi \& Vosátka, 2004). The industrial manufacturing of AMF as crop inoculants is relatively new, and, despite practical demonstrations of the efficiency of AMF, and crop producers have been slow to adopt them. Inoculation with effective microorganisms could lead to enhanced crop productivity and higher incomes for farmers.

The effectiveness of the AM symbiosis is highly dependent on the host plant genotype (Yücel et al., 2009). Differences in the level of association of AMF with particular genotypes were found in four Canadian spring wheat cultivars (Xavier \& Germida, 1998). These hexaploid Canadian wheat genotypes differed in AM root mycorrhizal development levels and in their response to inoculation, which ranged from positive to negative. The selection of crop cultivars that have strong associations with AMF may be important in providing adequate soil fertility to the crop (Singh et al., 2012). 
The aim of this study was therefore to evaluate the influence of AMF on biomass, root morphology and yield in spring wheat lines.

\section{Materials and Methods}

\subsection{Arbuscular Mycorrhizal Fungi Strain}

Two granular inoculants were applied at seeding as follows: $20 \mathrm{~g}$ of Rhizoglomus irregulare (MYKE PRO commercial inoculants; 1-propagule/g), or $1 \mathrm{~g}$ of Glomus cubense (living culture of the type-specimen DAOM $241198 ; 1000$-spores/g) per pot. Liquid G. cubense inoculant (20-spores $/ \mathrm{mL})$ obtained from the National Institute of Agricultural Science in Cuba (Fernández et al., 2004) was applied, through water irrigation, $7 \mathrm{~d}$ after seed germination $(25-\mathrm{mL}$ per pot). Controls plants were not inoculated.

\subsection{Experimental Design and Plant Material}

This experiment was conducted in a greenhouse at Agriculture and Agri-Food Canada (Ottawa Research and Development Centre), in Ottawa, Ontario, Canada, under controlled conditions during the period of February to June 2015. The experiment was performed in a completely randomized design with a split- plot arrangement. Five lines of spring wheat (Triticum aestivum L.) (AW-774, AC Carberry, HY-162, Major and AAC Scotia), two granular AMF strains ( $R$. irregulare and G. cubense), and liquid G. cubense mycorrhizal treatments were used. The main plot was AMF, and the subplot was spring wheat lines. Each treatment had six replicates for a total 120 pots. The soil used was sieved, homogenized, and sterilized (twice on successive days), and then mixed with pure washed sand $(1: 1, \mathrm{v} / \mathrm{v})$. Seeds were sown by hand at a depth of $2.5 \mathrm{~cm}$ after a germination test had been performed. Six seeds were sown per pot, and one month after planting, two plants per pot were removed. After seeding, irrigation with tap water was applied to maintain soil moisture near the maximum water-holding capacity. Urea was applied two times at a rate of 5 -g per pot after 30 days of plant growth.

\subsection{Measurements and Analysis}

At 120 days, two random test plants were uprooted carefully from each pot treatment. The roots were washed with tap water, and a fresh root portion (200 g) was used to estimate root colonization levels by the grid line intersect method (Giovannetti \& Mosse, 1980) after the roots had been bleached with $10 \% \mathrm{KOH}$ using the microwave oven (Dalpé \& Séguin, 2013) and stained with acid fuchsin (Phillips \& Hayman, 1970). The frequency and intensity of colonization indicators were determined according to the methodology described in Trouvelot et al. (1986).

Plant height, fresh and dry roots and plants biomasses were measured. Total length $(\mathrm{cm})$ and width $\left(\mathrm{cm}^{2}\right)$ of roots were measured using WinRHIZO Pro image analysis software. The number of grains per spike, number of grains per plant, grain weight, and yield were determined. Grain protein was evaluated using Kjeldahl digestion and total Kjeldahl nitrogen analysis.

\subsection{Statistical Analysis}

Data were analysed using the GLM procedure of the SAS software package (SAS Institute, 1989), and the means were separated using the least significant difference (LSD) method at the 5\% level.

\section{Results and Discussion}

The effects of mycorrhizal inoculation on different parameters of the spring wheat lines AW-774, AC Carberry, HY-162, Major, and AAC Scotia are shown in Tables 1 to 5, respectively. Mycorrhizal root colonization was different between spring wheat lines and between AMF treatments. The Major and AW-774 lines showed a higher level of colonization (58\% - 59\%), whereas AC Carberry and HY-162 showed a lower level (46\% - 47\%). The AAC Scotia line showed the lowest mycorrhizal colonization level $(35 \%)$ in comparison with two other wheat lines.

Arbuscular mycorrhizal fungi interact at the root-soil interface in a coordinated manner. Their hyphae absorb and translocate water and nutrients from the soil to the plant to increase its growth and development (Dwivedi, 2015; Priyadharsini \& Muthukumar, 2015). Wheat is colonized by mycorrhizae, but colonization depends on AMF strains and soil conditions. Studies conducted in India showed that the interaction between mycorrhizae and wheat depends on the variety, and thus it is important to evaluate mycorrhizal dependency in the crops being grown (Solaiman et al., 2014).

Table1. Effects of arbuscular mycorrhizal fungal inoculation of spring wheat line AW-774 on plant parameters.

\begin{tabular}{|c|c|c|c|c|c|c|c|c|c|c|c|c|}
\hline Treatment & $\begin{array}{l}\text { Yield } \\
(\mathrm{g} / \text { pot })\end{array}$ & $\begin{array}{l}\text { Plant } \\
\text { height } \\
(\mathrm{cm})\end{array}$ & $\begin{array}{l}\text { Spike } \\
\text { weight } \\
\text { (g) }\end{array}$ & $\begin{array}{l}\text { Tiller } \\
\text { number }\end{array}$ & $\begin{array}{l}\text { Fresh } \\
\text { biomass } \\
\text { (g) }\end{array}$ & $\begin{array}{l}\text { Dry } \\
\text { biomass } \\
\text { (g) }\end{array}$ & $\begin{array}{l}\text { Fresh root } \\
\text { biomass (g) }\end{array}$ & $\begin{array}{l}\text { Dry root } \\
\text { biomass }(\mathrm{g})\end{array}$ & $\begin{array}{l}\text { Root colonization } \\
\text { frequency }(\%)\end{array}$ & $\begin{array}{l}\text { Root } \\
\text { colonization } \\
\text { intensity }(\%)\end{array}$ & $\begin{array}{l}\text { Root total } \\
\text { length } \\
(\mathrm{cm})\end{array}$ & $\begin{array}{l}\text { Root total } \\
\text { width }\left(\mathrm{cm}^{2}\right)\end{array}$ \\
\hline Control & $4.77 \mathrm{c}$ & 50.87 & $6.52 \mathrm{c}$ & $6.33 \mathrm{c}$ & 1.21 & 1.19 & $14.55 \mathrm{c}$ & 0.40 & $1.00 \mathrm{c}$ & $0.01 \mathrm{c}$ & $413.50 \mathrm{~b}$ & $16.82 \mathrm{c}$ \\
\hline Glomus cubense (Liquid) & $7.70 \mathrm{a}$ & 57.47 & $9.73 \mathrm{a}$ & $9.67 \mathrm{a}$ & 1.30 & 1.29 & $23.72 \mathrm{a}$ & 0.47 & $58.00 \mathrm{a}$ & $2.45 \mathrm{a}$ & $923.30 \mathrm{a}$ & $42.52 \mathrm{a}$ \\
\hline $\begin{array}{l}\text { Rhizoglomus irregulare } \\
\text { (Solid) }\end{array}$ & $6.75 \mathrm{~b}$ & 52.88 & $8.55 \mathrm{~b}$ & $7.50 \mathrm{~b}$ & 1.23 & 1.23 & $17.58 \mathrm{~b}$ & 0.44 & $35.50 \mathrm{~b}$ & $0.58 \mathrm{~b}$ & $836.90 \mathrm{a}$ & $37.23 \mathrm{a}$ \\
\hline Glomus cubense (Solid) & $8.48 \mathrm{a}$ & 57.25 & $10.67 \mathrm{a}$ & $9.67 \mathrm{a}$ & 1.33 & 1.30 & $23.19 \mathrm{a}$ & 0.42 & $59.17 \mathrm{a}$ & $2.48 \mathrm{a}$ & $592.50 \mathrm{~b}$ & $30.06 \mathrm{ab}$ \\
\hline$F$-value & 27.94 & 1.52 & 30.45 & 28.67 & 1.54 & 1.45 & 61.50 & 1.40 & 379.39 & 66.70 & 9.15 & 10.22 \\
\hline LSD & 0.89 & $7.82 \mathrm{NS}$ & 0.96 & 0.91 & $0.13 \mathrm{NS}$ & $0.13 \mathrm{NS}$ & 1.67 & $0.07 \mathrm{NS}$ & 4.13 & 0.46 & 226.59 & 10.27 \\
\hline SE & 0.30 & 2.65 & 0.32 & 0.31 & 0.04 & 0.04 & 0.57 & 0.02 & 1.40 & 0.16 & 76.81 & 3.48 \\
\hline
\end{tabular}

Note: Different letters within each block indicate significant differences $(p \leq 0.05)$ according to the least significant difference (LSD) test. NS: not significant.

SE: standard error 
Table 2. Effects of arbuscular mycorrhizal fungal inoculation of spring wheat line AC Carberry on plant parameters.

\begin{tabular}{|c|c|c|c|c|c|c|c|c|c|c|c|c|}
\hline Treatment & $\begin{array}{l}\text { Yield } \\
\text { (g/pot) }\end{array}$ & $\begin{array}{c}\text { Plant } \\
\text { height } \\
(\mathrm{cm})\end{array}$ & $\begin{array}{l}\text { Spike } \\
\text { weight } \\
\text { (g) }\end{array}$ & $\begin{array}{l}\text { Tiller } \\
\text { number }\end{array}$ & $\begin{array}{l}\text { Fresh } \\
\text { biomass } \\
\text { (g) }\end{array}$ & $\begin{array}{l}\text { Dry } \\
\text { biomass } \\
\text { (g) }\end{array}$ & $\begin{array}{c}\text { Fresh } \\
\text { root } \\
\text { biomass } \\
\text { (g) } \\
\end{array}$ & $\begin{array}{l}\text { Dry root } \\
\text { biomass } \\
\text { (g) }\end{array}$ & $\begin{array}{c}\begin{array}{c}\text { Root } \\
\text { colonization } \\
\text { frequency } \\
(\%)\end{array} \\
\end{array}$ & $\begin{array}{c}\text { Root } \\
\text { colonization } \\
\text { intensity }(\%)\end{array}$ & $\begin{array}{c}\text { Root } \\
\text { total } \\
\text { length } \\
(\mathrm{cm}) \\
\end{array}$ & $\begin{array}{l}\text { Root } \\
\text { total } \\
\text { width } \\
\left(\mathrm{cm}^{2}\right) \\
\end{array}$ \\
\hline Control & $3.66 \mathrm{~b}$ & $42.83 \mathrm{~b}$ & $4.96 \mathrm{~b}$ & $6.33 \mathrm{c}$ & $0.90 \mathrm{~b}$ & $0.87 \mathrm{~b}$ & $11.05 \mathrm{~d}$ & $0.25 \mathrm{c}$ & $1.00 \mathrm{c}$ & $0.01 \mathrm{~d}$ & $391.55 \mathrm{~b}$ & $16.51 \mathrm{~b}$ \\
\hline $\begin{array}{l}\text { Glomus cubense } \\
\text { (Liquid) }\end{array}$ & $5.22 \mathrm{a}$ & $49.40 \mathrm{a}$ & $7.06 \mathrm{a}$ & $10.17 \mathrm{a}$ & $1.17 \mathrm{a}$ & $1.15 \mathrm{a}$ & $19.52 \mathrm{~b}$ & $0.34 \mathrm{a}$ & $47.50 \mathrm{a}$ & $2.23 \mathrm{a}$ & $383.84 \mathrm{~b}$ & $15.89 \mathrm{~b}$ \\
\hline $\begin{array}{l}\text { Rhizoglomus } \\
\text { irregulare (Solid) }\end{array}$ & $4.46 \mathrm{ab}$ & $44.12 \mathrm{~b}$ & $6.35 \mathrm{a}$ & $7.00 \mathrm{c}$ & $1.05 \mathrm{ab}$ & $1.02 \mathrm{ab}$ & $14.16 \mathrm{c}$ & $0.31 \mathrm{~b}$ & $36.67 \mathrm{~b}$ & $0.60 \mathrm{c}$ & $494.60 \mathrm{a}$ & $23.26 \mathrm{a}$ \\
\hline $\begin{array}{l}\text { Glomus cubense } \\
\text { (Solid) }\end{array}$ & $5.22 \mathrm{a}$ & $49.43 \mathrm{a}$ & $7.12 \mathrm{a}$ & $8.67 \mathrm{a}$ & $1.19 \mathrm{a}$ & $1.16 \mathrm{a}$ & $20.89 \mathrm{a}$ & $0.37 \mathrm{a}$ & $46.83 \mathrm{a}$ & $1.83 \mathrm{~b}$ & $559.05 \mathrm{a}$ & $26.33 \mathrm{a}$ \\
\hline$F$-value & 6.26 & 6.74 & 7.51 & 20.37 & 5.24 & 5.67 & 125.50 & 12.25 & 369.87 & 99.28 & 7.10 & 5.14 \\
\hline LSD & 0.88 & 3.94 & 1.08 & 1.13 & 0.17 & 0.17 & 1.21 & 0.04 & 3.36 & 0.31 & 93.67 & 6.67 \\
\hline SE & 0.30 & 1.34 & 0.37 & 0.38 & 0.06 & 0.06 & 0.41 & 0.01 & 1.14 & 0.10 & 31.75 & 2.26 \\
\hline
\end{tabular}

Note: Different letters within each block indicate significant differences $(\mathrm{p} \leq 0.05)$ according to the least significant difference (LSD) test. NS: not significant.

SE: standard error

Table 3. Effects of arbuscular mycorrhizal fungal inoculation of spring wheat line HY-162 on plant parameters.

\begin{tabular}{|c|c|c|c|c|c|c|c|c|c|c|c|c|}
\hline Treatment & $\begin{array}{l}\text { Yield } \\
\text { (g/pot) }\end{array}$ & $\begin{array}{l}\text { Plant } \\
\text { height } \\
(\mathbf{c m})\end{array}$ & $\begin{array}{l}\text { Spike } \\
\text { weight } \\
\text { (g) }\end{array}$ & $\begin{array}{l}\text { Tiller } \\
\text { number }\end{array}$ & $\begin{array}{l}\text { Fresh } \\
\text { biomass } \\
\text { (g) }\end{array}$ & $\begin{array}{l}\text { Dry } \\
\text { biomass } \\
\text { (g) }\end{array}$ & $\begin{array}{l}\text { Fresh } \\
\text { root } \\
\text { biomass } \\
\text { (g) } \\
\end{array}$ & $\begin{array}{l}\text { Dry root } \\
\text { biomass } \\
\text { (g) }\end{array}$ & $\begin{array}{l}\text { Root } \\
\text { colonization } \\
\text { frequency }(\%)\end{array}$ & $\begin{array}{l}\text { Root } \\
\text { colonization } \\
\text { intensity (\%) }\end{array}$ & $\begin{array}{l}\text { Root total } \\
\text { length } \\
(\mathrm{cm})\end{array}$ & $\begin{array}{l}\text { Root } \\
\text { total } \\
\text { width } \\
\left(\mathrm{cm}^{2}\right)\end{array}$ \\
\hline Control & $3.48 \mathrm{~b}$ & $31.35 \mathrm{c}$ & $4.79 \mathrm{~b}$ & $6.33 \mathrm{~b}$ & $0.75 \mathrm{~b}$ & $0.74 \mathrm{~b}$ & $9.53 b$ & $0.19 \mathrm{~b}$ & $1.00 \mathrm{c}$ & $0.01 \mathrm{~d}$ & 348.75 & 16.12 \\
\hline $\begin{array}{l}\text { Glomus cubense } \\
\text { (Liquid) }\end{array}$ & $4.70 \mathrm{a}$ & $42.10 \mathrm{a}$ & $5.90 \mathrm{a}$ & $8.33 \mathrm{a}$ & $0.98 \mathrm{a}$ & $0.96 \mathrm{a}$ & $13.71 \mathrm{a}$ & $0.26 \mathrm{a}$ & $45.67 \mathrm{a}$ & $6.52 \mathrm{a}$ & 384.23 & 18.82 \\
\hline $\begin{array}{l}\text { Rhizoglomus } \\
\text { irregulare (Solid) }\end{array}$ & $3.68 \mathrm{~b}$ & $36.50 \mathrm{~b}$ & $5.01 \mathrm{~b}$ & $6.33 \mathrm{~b}$ & $0.87 \mathrm{ab}$ & $0.85 \mathrm{ab}$ & $10.25 \mathrm{~b}$ & $0.24 \mathrm{a}$ & $36.50 \mathrm{~b}$ & $0.60 \mathrm{c}$ & 390.50 & 15.82 \\
\hline $\begin{array}{l}\text { Glomus cubense } \\
\text { (Solid) }\end{array}$ & $4.64 \mathrm{a}$ & $42.03 \mathrm{a}$ & $5.94 \mathrm{a}$ & $8.50 \mathrm{a}$ & $1.00 \mathrm{a}$ & $0.98 \mathrm{a}$ & $13.05 \mathrm{a}$ & $0.24 \mathrm{ab}$ & $47.50 \mathrm{a}$ & $2.23 \mathrm{~b}$ & 354.38 & 16.43 \\
\hline$F$-value & 7.10 & 15.87 & 5.49 & 18.33 & 4.26 & 3.78 & 20.11 & 2.67 & 519.32 & 1.56 & 0.46 & 0.92 \\
\hline LSD & 0.71 & 3.81 & 0.75 & 0.83 & 0.16 & 0.17 & 1.35 & 0.05 & 2.80 & 6.93 & $90.78 \mathrm{NS}$ & $4.23 \mathrm{NS}$ \\
\hline SE & 0.24 & 1.29 & 0.25 & 0.28 & 0.06 & 0.06 & 0.46 & 0.02 & 0.95 & 2.35 & 30.67 & 1.44 \\
\hline
\end{tabular}

Note: Different letters within each block indicate significant differences $(p \leq 0.05)$ according to the least significant difference (LSD) test. NS: not significant.

SE: standard error

Table 4. Effects of arbuscular mycorrhizal fungal inoculation of spring wheat line Major on plant parameters.

\begin{tabular}{|c|c|c|c|c|c|c|c|c|c|c|c|c|}
\hline Treatment & $\begin{array}{l}\text { Yield } \\
\text { (g/pot) }\end{array}$ & $\begin{array}{l}\text { Plant } \\
\text { height } \\
(\mathrm{cm})\end{array}$ & $\begin{array}{l}\text { Spike } \\
\text { weight } \\
\text { (g) }\end{array}$ & $\begin{array}{l}\text { Tiller } \\
\text { number }\end{array}$ & $\begin{array}{l}\text { Fresh } \\
\text { biomass } \\
\text { (g) }\end{array}$ & $\begin{array}{l}\text { Dry } \\
\text { biomass } \\
\text { (g) }\end{array}$ & $\begin{array}{l}\text { Fresh } \\
\text { root } \\
\text { biomass } \\
\text { (g) }\end{array}$ & $\begin{array}{l}\text { Dry root } \\
\text { biomass } \\
\text { (g) }\end{array}$ & $\begin{array}{l}\text { Root } \\
\text { colonization } \\
\text { frequency } \\
(\%)\end{array}$ & $\begin{array}{l}\text { Root } \\
\text { colonization } \\
\text { intensity }(\%)\end{array}$ & $\begin{array}{l}\text { Root total } \\
\text { length } \\
(\mathrm{cm})\end{array}$ & $\begin{array}{l}\text { Root } \\
\text { total } \\
\text { width } \\
\left(\mathrm{cm}^{2}\right)\end{array}$ \\
\hline Control & $4.73 \mathrm{c}$ & $50.21 \mathrm{c}$ & $7.09 \mathrm{c}$ & $6.17 \mathrm{~b}$ & $1.31 \mathrm{~b}$ & $1.28 \mathrm{~b}$ & $15.60 \mathrm{c}$ & $0.41 \mathrm{c}$ & $1.00 \mathrm{c}$ & $0.01 \mathrm{c}$ & $433.06 \mathrm{c}$ & $17.18 \mathrm{c}$ \\
\hline $\begin{array}{l}\text { Glomus cubense } \\
\text { (Liquid) }\end{array}$ & $7.45 \mathrm{a}$ & $79.37 \mathrm{a}$ & $9.78 \mathrm{a}$ & $9.17 \mathrm{a}$ & $1.59 \mathrm{a}$ & $1.58 \mathrm{a}$ & $36.25 \mathrm{a}$ & $0.73 \mathrm{a}$ & $58.17 \mathrm{a}$ & $2.47 \mathrm{a}$ & $988.21 \mathrm{a}$ & $43.00 \mathrm{a}$ \\
\hline $\begin{array}{l}\text { Rhizoglomus } \\
\text { irregulare (Solid) }\end{array}$ & $6.38 \mathrm{~b}$ & $56.90 \mathrm{~b}$ & $8.42 \mathrm{~b}$ & $6.83 \mathrm{~b}$ & $1.39 \mathrm{~b}$ & $1.37 \mathrm{~b}$ & $24.46 \mathrm{~b}$ & $0.47 \mathrm{~b}$ & $38.33 \mathrm{~b}$ & $0.79 \mathrm{~b}$ & $736.96 \mathrm{~b}$ & $33.76 \mathrm{~b}$ \\
\hline $\begin{array}{l}\text { Glomus cubense } \\
\text { (Solid) }\end{array}$ & $7.87 \mathrm{a}$ & $79.62 \mathrm{a}$ & $9.60 \mathrm{a}$ & 10. $17 \mathrm{a}$ & $1.60 \mathrm{a}$ & $1.59 \mathrm{a}$ & $35.95 \mathrm{a}$ & $0.74 \mathrm{a}$ & $58.00 \mathrm{a}$ & $2.45 \mathrm{a}$ & $1010.02 \mathrm{a}$ & $48.04 \mathrm{a}$ \\
\hline$F$-value & 16.10 & 119.38 & 10.59 & 28.04 & 11.99 & 11.95 & 163.71 & 154.00 & 429.18 & 48.11 & 31.24 & 47.05 \\
\hline LSD & 1.03 & 4.11 & 1.13 & 1.05 & 0.12 & 0.13 & 2.29 & 0.04 & 3.87 & 0.52 & 142.25 & 5.83 \\
\hline SE & 0.35 & 1.39 & 0.38 & 0.36 & 0.04 & 0.04 & 0.78 & 0.01 & 1.31 & 0.18 & 48.22 & 1.98 \\
\hline
\end{tabular}

Note: Different letters within each block indicate significant differences $(\mathrm{p} \leq 0.05)$ according to the least significant difference (LSD) test. SE: standard error

Table 5. Effects of arbuscular mycorrhizal fungal inoculation of spring wheat line AC Scotia on plant parameters.

\begin{tabular}{|c|c|c|c|c|c|c|c|c|c|c|c|c|}
\hline Treatment $^{\mathrm{a}}$ & $\begin{array}{l}\text { Yield } \\
\text { (g/pot) }\end{array}$ & $\begin{array}{l}\text { Plant } \\
\text { height } \\
(\mathbf{c m})\end{array}$ & $\begin{array}{l}\text { Spike } \\
\text { weight } \\
\text { (g) }\end{array}$ & $\begin{array}{l}\text { Tiller } \\
\text { number }\end{array}$ & $\begin{array}{l}\text { Fresh } \\
\text { biomass } \\
\text { (g) }\end{array}$ & $\begin{array}{l}\text { Dry } \\
\text { biomass } \\
\text { (g) }\end{array}$ & $\begin{array}{l}\text { Fresh } \\
\text { root } \\
\text { biomass } \\
\text { (g) } \\
\end{array}$ & $\begin{array}{l}\text { Dry root } \\
\text { biomass } \\
\text { (g) }\end{array}$ & $\begin{array}{l}\text { Root } \\
\text { colonization } \\
\text { frequency } \\
(\%) \\
\end{array}$ & $\begin{array}{l}\text { Root } \\
\text { colonization } \\
\text { intensity (\%) }\end{array}$ & $\begin{array}{l}\text { Root } \\
\text { total } \\
\text { length } \\
(\mathrm{cm}) \\
\end{array}$ & $\begin{array}{l}\text { Root } \\
\text { total } \\
\text { width } \\
(\mathrm{cm}) \\
\end{array}$ \\
\hline Control & $5.28 \mathrm{~b}$ & 40.67 & $7.81 \mathrm{~b}$ & 5.50 & 1.21 & 1.19 & $8.73 \mathrm{c}$ & $0.21 \mathrm{~b}$ & $1.00 \mathrm{~b}$ & $0.01 \mathrm{~b}$ & $403.75 \mathrm{ab}$ & $16.73 \mathrm{ab}$ \\
\hline $\begin{array}{l}\text { Glomus cubense } \\
\text { (Liquid) }\end{array}$ & $6.08 \mathrm{a}$ & 41.87 & $9.09 \mathrm{a}$ & 5.67 & 1.22 & 1.20 & $12,05 \mathrm{a}$ & $0.25 \mathrm{a}$ & $35.66 \mathrm{a}$ & $0.58 \mathrm{a}$ & $488.80 \mathrm{a}$ & $23.44 a$ \\
\hline $\begin{array}{l}\text { Rhizoglomus } \\
\text { irregulare (Solid) }\end{array}$ & $5.62 \mathrm{ab}$ & 41.00 & $7.69 \mathrm{~b}$ & 5.33 & 1.19 & 1.17 & $10.63 \mathrm{~b}$ & $0.22 \mathrm{ab}$ & $35.50 \mathrm{a}$ & $0.56 \mathrm{a}$ & $485.05 \mathrm{a}$ & $21.66 \mathrm{ab}$ \\
\hline $\begin{array}{l}\text { Glomus ubense } \\
\text { (Solid) }\end{array}$ & $5.98 \mathrm{ab}$ & 40.80 & $8.22 \mathrm{ab}$ & 5.50 & 1.21 & 1.19 & $12.43 \mathrm{a}$ & $0.24 \mathrm{ab}$ & $36.50 \mathrm{a}$ & $0.59 \mathrm{a}$ & $285.10 \mathrm{~b}$ & $15.55 \mathrm{~b}$ \\
\hline$F$-value & 1.91 & 0.19 & 3.82 & 0.39 & 0.05 & 0.05 & 15.25 & 1.86 & 298.69 & 197.06 & 3.79 & 2.65 \\
\hline LSD & 0.77 & $3.67 \mathrm{NS}$ & 0.95 & $0.64 \mathrm{NS}$ & $0.13 \mathrm{NS}$ & $0.14 \mathrm{NS}$ & 1.26 & 0.04 & 2.98 & 0.06 & 144.69 & 6.90 \\
\hline SE & 0.26 & 1.24 & 0.32 & 0.22 & 0.05 & 0.05 & 0.43 & 0.01 & 1.01 & 0.02 & 49.05 & 2.34 \\
\hline
\end{tabular}

Note: Different letters within each block indicate significant differences $(\mathrm{p} \leq 0.05)$ according to the least significant difference (LSD) test. NS: not significant.

SE: standard error 
The mycorrhizal colonization results showed differences between G.cubense and R.irregulare, which could be related to infectivity. Some studies demonstrated that fungal infectivity can be associated with differences in inoculum level and in the ability of the fungi to colonize roots (Solaiman et al., 2014). In our case, the inoculum concentration was different for both strains; such a difference can induce variations in fungal colonization and interfere with the relationship between inoculum level and infectivity. However, other research demonstrated a positive effect of the G.cubense strain in a different crop grown in red soil with low to high fertility (Rivera et al., 2007). The mycorrhizal colonization levels obtained in the present study are similar to those reported for rice cultivation in saline conditions (Fernández et al., 2011).

The root morphology results showed significant differences between wheat lines, but for the HY-162 line, no positive effect to AMF was found. A comprehensive analysis of root morphology variables for the Major wheat line showed that G.cubense was more effective than R.irregulare. The results for these variables were in agreement with those for fungal colonization. Although root morphological variables for the AAC Scotia line showed significant differences, the level of mycorrhizal colonization was low, with no significant differences from the levels for the other lines, and exceeded only the level for the control treatment.

Significant differences in plant growth indicators (plant height, fresh and dry weights of roots and biomass) between all five wheat lines under study were determinated. In two lines (AW-774 and AAC Scotia), inoculation with AMF strains showed no positive effect on plant height, but in the rest of the lines, significant differences were obtained in relation to the control treatment.

The tallest plants were achieved with the Major wheat line, at $79 \mathrm{~cm}$, in the treatments inoculated with G.cubense, whereas inoculation of Major with R.irregulare produced a height of $56 \mathrm{~cm}$. Inoculation with liquid and solid G.cubense stimulated plant height for the AC Carberry and HY-162 wheat lines, with values of 49 and $42 \mathrm{~cm}$, respectively. Root dry weight differed between wheat lines and between inoculation treatments. The AW-774 wheat line did not show significant differences for this indicator, but levels were variable and exceeded the value for the control treatment.

Plant growth indicators can be used to evaluate benefits when microorganisms are applied, given that plant growth and development are stimulated significantly. Our results show differences depending on the AMF strains and spring wheat lines, but inoculant treatment responded to fungal colonization. The results were higher when G.cubense (liquid and solid) inoculum was applied. This response could be related to mycorrhizal effectiveness (Bonfante \& Genre, 2010; Fernández et al., 2011). The positive effect of AMF on the height and development of the aerial part and root system of plants was previouly reported in various crops, including maize (Zea mays) (Sheng et al., 2011), tomato (Solanum lycopersicum) (Hajiboland et al., 2010), wheat (Triticum aestivum) (Stonor et al., 2014), rice (Oryza sativa) (Fernández et al., 2011) and pepper (Capsicum annuum) (Çekiç et al., 2012).

The yield of a crop is the end result of the interaction of several factors. In this study, differences between AMF strains and between wheat lines were found for yield components. Inoculation with liquid and solid G.cubense increased tiller number and spike weight in four of the wheat lines (AW-774, AC Carberry, HY-162, and Major). Inoculation with R.irregulare increased the levels of those indicators as well, but to a lower extent than inoculation with G.cubense did. Wheat yield was stimulated by AMF inoculation. Inoculation with liquid and solid G.cubense was more effective than inoculation with R.irregulare was, but both species showed better results than control treatment.

The effects of mycorrhizal inoculation on total nitrogen and grain protein in the spring wheat lines are shown in Table -6. In this case, AMF inoculation did not have a positive effect for both indicators, and only the HY-162 line showed significant differences. This response could be related to the fertilization and mineral requirements of each wheat line.

Table 6. Effects of arbuscular mycorrhizal fungal inoculation of spring wheat lines on total nitrogen $(\mathrm{N})$ and grain protein.

\begin{tabular}{|c|c|c|c|c|c|c|c|c|c|c|}
\hline \multirow[t]{2}{*}{ Treatment $^{\mathrm{a}}$} & \multicolumn{2}{|c|}{ AW-774 } & \multicolumn{2}{|c|}{ AC Carberry } & \multicolumn{2}{|c|}{ HY-162 } & \multicolumn{2}{|r|}{ Major } & \multicolumn{2}{|c|}{ AAC Scotia } \\
\hline & $\begin{array}{c}\text { Total N } \\
(\%)\end{array}$ & $\begin{array}{c}\text { Grain } \\
\text { protein (\%) }\end{array}$ & $\begin{array}{c}\text { Total N } \\
(\%)\end{array}$ & $\begin{array}{c}\text { Grain } \\
\text { protein }(\%)\end{array}$ & $\begin{array}{c}\text { Total N } \\
(\%)\end{array}$ & $\begin{array}{c}\text { Grain } \\
\text { protein }(\%)\end{array}$ & $\begin{array}{c}\text { Total N } \\
(\%)\end{array}$ & $\begin{array}{c}\text { Grain } \\
\text { protein }(\%)\end{array}$ & $\begin{array}{c}\text { Total N } \\
(\%)\end{array}$ & $\begin{array}{c}\text { Grain } \\
\text { protein }(\%)\end{array}$ \\
\hline Control & 3.27 & 18.63 & $3.50 \mathrm{~b}$ & 20.03 & $3.87 \mathrm{ab}$ & $22.10 \mathrm{ab}$ & 3.80 & 21.66 & 3.51 & 19.96 \\
\hline $\begin{array}{l}\text { Glomus cubense } \\
\text { (Liquid) }\end{array}$ & 3.27 & 18.63 & $3.70 \mathrm{a}$ & 21.10 & $3.98 \mathrm{a}$ & $22.70 \mathrm{a}$ & 3.67 & 21.00 & 3.70 & 21.10 \\
\hline $\begin{array}{l}\text { Rhizoglomus } \\
\text { irregulare (Solid) }\end{array}$ & 3.29 & 18.73 & $3.60 \mathrm{ab}$ & 20.53 & $3.60 \mathrm{c}$ & $20.56 \mathrm{c}$ & 3.37 & 19.20 & 3.60 & 20.53 \\
\hline $\begin{array}{l}\text { Glomus cubense } \\
\text { (Solid) }\end{array}$ & 3.43 & 19.56 & $3.64 \mathrm{a}$ & 20.80 & $3.68 \mathrm{bc}$ & $21.00 \mathrm{bc}$ & 3.49 & 19.90 & 3.45 & 19.70 \\
\hline$F$-value & 0.61 & 0.62 & 2.45 & 2.24 & 6.88 & 6.94 & 1.78 & 1.77 & 0.39 & 0.37 \\
\hline LSD & $0.32 \mathrm{NS}$ & $1.87 \mathrm{NS}$ & 0.17 & $0.98 \mathrm{NS}$ & 0.21 & 1.21 & $0.46 \mathrm{NS}$ & $2.70 \mathrm{NS}$ & $0.58 \mathrm{NS}$ & $3.35 \mathrm{NS}$ \\
\hline SE & 0.10 & 0.58 & 0.05 & 0.30 & 0.07 & 0.37 & 0.14 & 0.83 & 0.18 & 1.03 \\
\hline
\end{tabular}

Note: Different letters within each block indicate significant differences $(p \leq 0.05)$ according to the least significant difference (LSD) test. NS: not significant. SE: standard error

The results showed a positive effect of inoculation in comparison with the control treatment. Liquid and solid G.cubense inoculant produced better results than inoculation with R.irregulare. Fungus indicators were in 
agreement with root morphological parameters because of the effect induced by AMF activity. Yield increased significantly in the mycorrhizal treatments.

\section{Acknowledgements}

This work was supported by a Government of Canada international scholarship (Emerging Leaders in the Americas Program). The authors would like to acknowledge Agriculture and Agri-Food Canada greenhouse staff members Art Smith and Kathie Upton for their help maintaining the set-up.

\section{References}

Adesemoye, A. O., \& Kloepper, J. W. (2009). Plant-microbes interactions in enhanced fertilizer-use efficiency. Applied Microbiology and Biotechnology, 85, 1-12. http://dx.doi.org/10.1007/s00253-009-2196-0

Banuelos, J., Alarcón, A., Larsen, J., Cruz-Sánchez, S., \& Trejo, D. (2014). Interactions between arbuscular mycorrhizal fungi and Meloidogyne incognita in the ornamental plant Impatiens balsamina. Journal of Soil Science and Plant Nutrition, 14, 63-74.

Bonfante, P., \& Genre, A. (2010). Mechanisms underlying beneficial plant-fungus interactions in mycorrhizal symbiosis. Nature Communications, 1, 48. http://dx.doi.org/10.1038/ncomms1046

Çekiç, F. Ö., Ünyayar, S., \& Ortaş, I. (2012). Effects of arbuscular mycorrhizal inoculation on biochemical parameters in Capsicum annuum grown under long term salt stress. Turkish Journal of Botany, 36, 63-72.

Cicatelli, A., Todeschini, V., Lingua, G., Biondi, S., Torrigiani, P., \& Castiglione, S. (2014). Epigenetic control of heavy metal stress response in mycorrhizal versus non-mycorrhizal poplar plants. Environmental Science and Pollution Research, 21, 1723-1737. http://dx.doi.org/10.1007/s11356-013-2072-4

Dalpé, Y., \& Séguin, S. M. (2013). Microwave assisted technology for the clearing and staining of arbuscular mycorrhizal fungi in roots. Mycorrhiza, 23, 333-340. http://dx.doi.org/10.1007/s00572-012-0472-9

Dwivedi, O. P. (2015). Distribution and association of arbuscular mycorrhizal fungi in different cultivars of wheat from Lalganj Pratapgarh District of Utter Pradesh, India. Advances in Bioscience and Biotechnology, 6, 353-357. http://dx.doi.org/10.4236/abb.2015.65034

Fedoroff, N. V., Battisti, D. S., Beachy, R. N., Cooper, P. J. M., Fischhoff, D. A., Hodges, C. N., \& Zhu, J. K. (2010). Radically rethinking agriculture for the 21st century. Science, 327, 833-834. http://dx.doi.org/10.1126/science.1186834

Fernández, F., Dell'Amico, J. M., Angoa, M. V., \& de la Providencia, I. E. (2011). Use of a liquid inoculum of the arbuscular mycorrhizal fungi Glomus hoi in rice plants cultivated in a saline Gleysol: A new alternative to inoculate. Journal of Plant Breeding and Crop Science, 3, 24-33.

Fernández, F., Dell'Amico, J. M., \& Pérez, Y. (2004). Producto inoculante micorrizógeno líquido. Cuban Patent 23479.

Garnett, T., Appleby, M. C., Balmford, A., Bateman, I. J., Benton, T. G., Bloomer, P., \& Godfray, H. C. J. (2013). Sustainable intensification in agriculture: Premises and policies. Science, 341, 33-34. http://dx.doi.org/10.1126/science.1234485

Gianinazzi, S., \& Vosátka, M. (2004). Inoculum of arbuscular mycorrhizal fungi for production systems: science meets business. Canadian Journal of Botany, 82, 1264-1271. http://dx.doi.org/10.1139/b04-072

Giovannetti, M., \& Mosse, B. (1980). An evaluation of techniques for measuring vesicular-arbuscular mycorrhizal infection in roots. New Phytologist, 84, http://dx.doi.org/10.1111/j.1469-8137.1980.tb04556.x

Graham, J. H. (2008). Scaling-up evaluation of field functioning of arbuscular mycorrhizal fungi. New Phytologist, 180, 1-2. http://dx.doi.org/10.1111/j.1469-8137.2008.02608.x

Hajiboland, R., Aliasgharzadeh, N., Laiegh, S. F., \& Poschenrieder, C. (2010). Colonization with arbuscular mycorrhizal fungi improves salinity tolerance of tomato (Solanum lycopersicum L.) plants. Plant and Soil, 331, 313-327. http://dx.doi.org/10.1007/s11104-009-0255-z

Mrnka, L., Kuchár, M., Cieslarová, Z., Matějka, P., Száková, J., Tlustoš, P., \& Vosátka, M. (2012). Effects of endoand ectomycorrhizal fungi on physiological parameters and heavy metals accumulation of two species from the family Salicaceae. Water, Air and Soil Pollution, 223, 399-410. http://dx.doi.org/10.1007/s11270-011-0868-8

Mueller, N. D., Gerber, J. S., Johnston, M., Ray, D. K., Ramankutty, N., \& Foley, J. A. (2012). Closing yield gaps through nutrient and water management. Nature, 490, 254-257. http://dx.doi.org/10.1038/nature11420

Neumann, E., \& George, E. (2010). Nutrient uptake: The arbuscular mycorrhiza fungal symbiosis as a plant nutrient acquisition strategy. In: H. Koltai \& Y. Kapulnik (Eds) Arbuscular mycorrhizas: Physiology and function (pp. 137-167). Dordrecht, Netherlands: Springer. http://dx.doi.org/10.1007/978-90-481-9489-6_7

Priyadharsini, P., \& Muthukumar, T. (2015). Insight into the role of arbuscular mycorrhizal fungi in sustainable agriculture. In: P. Thangavel \& G. Sridevi (Eds), Environmental sustainability: Role of green technologies (pp. 3-37). New Delhi:Springer. http://dx.doi.org/10.1007/978-81-322-2056-5_1

Rivera, R., Fernández, F., Fernández, K., Ruiz, L., Sánchez, C., \& Riera, M. (2007). Advances in the management 
of effective arbuscular mycorrhizal symbiosis in tropical ecosystems. In: C. Hamel \& C. Plenchette (Eds), Mycorrhizae in crop production (pp. 151-188). Binghampton, NY: Haworth Press.

Ruiz-Lozano, J. M., Porcel, R., Azcón, C., \& Aroca, R. (2012). Regulation by arbuscular mycorrhizae of the integrated physiological response to salinity in plants: New challenges in physiological and molecular studies. Journal of Experimental Botany, 63, 4033-4044. http://dx.doi.org/10.1093/jxb/ers126

SAS, Institute. (1989). SAS user's guide: Statistics. Version 6. SAS Institute Inc., Cary, NC, USA.

Sheng, M., Tang, M., Zhang, F., \& Huang, Y. (2011). Influence of arbuscular mycorrhiza on organic solutes in maize leaves under salt stress. Mycorrhiza, 21, 423-430. http://dx.doi.org/10.1007/s00572-010-0353-z

Singh, A.K., Hamel, C., DePauw, R.M., \& Knox, R.E. (2012). Genetic variability in arbuscular mycorrhizal fungi compatibility supports the selection of durum wheat genotypes for enhancing soil ecological services and cropping systems in Canada. Canadian Journal of Microbiology, 58, 293-302. http://dx.doi.org/10.1139/w11-140

Smith, S. E., \& Read, D. J. (2008). Mycorrhizal symbiosis. (3rd ed.). Academic Press: New York.

Solaiman, Z. M., Abbott, L. K., \& Varma, A. (Eds.). (2014). Mycorrhizal fungi: Use in sustainable agriculture and land restoration. Berlin: Springer.

Songachan, L. S., Lyngdoh, I., \& Highland, K. (2011). Colonization of arbuscular mycorrhizal fungi in moderately degraded sub-tropical forest stands of Meghalaya, Northeast India. Journal of Agricultural Technology, 7, 1673-1684.

Stonor, R. N., Smith, S. E., Manjarrez, M., Facelli, E., \& Smith, A. (2014). Mycorrhizal responses in wheat: Shading decreases growth but does not lower the contribution of the fungal phosphate uptake pathway. Mycorrhiza, 24, 465-472. http://dx.doi.org/10.1007/s00572-014-0556-9

Tilman, D., Balzer, C., Hill, J., \& Befort, B. L. (2011). Global food demand and the sustainable intensification of agriculture. Proceedings of the National Academy of Science, 108, 20260-20264. http://dx.doi.org/10.1073/pnas.1116437108

Trouvelot, A., Kough, J., \& GianinazziPearson, V. (1986). Mesure du taux de mycorhization VA d'un systeme radiculaire. Recherche de méthodes d'estimation ayantune signification fonctionnelle. In: V. Gianinazzi-Pearson \& S. Gianinazzi (Eds.), Physiological and genetical aspects of mycorrhizae: Proceedings of the 1st European Symposium on Mycorrhizae (pp. 217-222). Paris: INRA

Xavier, L. J. C., \& Germida, J. J. (1998). Response of spring wheat cultivars to Glomus clarum NT4 in a P-deficient soil containing arbuscular mycorrhizal fungi. Canadian Journal of Soil Science, 78, 481-484. http://dx.doi.org/10.4141/S97-106

Yücel, C., Özkan, H., Ortaş , I., \& Yağbasanlar, T. (2009). Screening of wild emmer wheat accessions (Triticum turgidum subsp. dicoccoides) for mycorrhizal dependency. Turkish Journal of Agriculture and Forestry, 33, 513-523.

\section{Copyrights}

Copyright for this article is retained by the author(s), with first publication rights granted to the journal.

This is an open-access article distributed under the terms and conditions of the Creative Commons Attribution license (http://creativecommons.org/licenses/by/4.0/). 